\title{
STUDY OF STRUCTURAL AND MECHANICAL PROPERTIES OF SODIUM ALGINATE GELS
}

\author{
Ievgenii Gladukh \\ Department of Pharmaceutical Technology of Drugs ${ }^{1}$ \\ glad_e@i.ua \\ Maiia Podorozhna \\ Department of Pharmaceutical Technology of Drugs ${ }^{l}$ \\ uglavamaya@gmail.com \\ ${ }^{I}$ National University of Pharmacy \\ 53 Pushkinska str., Kharkiv, Ukraine, 61002
}

\begin{abstract}
Rheological parameters are an important characteristic of semisolid dosage forms. Structural and mechanical characteristics have a noticeable effect on the processes of release and absorption of drugs from ointments, as well as on their consumer properties: spreadability, adhesion, the ability to squeeze out of the tubes.

The aim: to study the rheological parameters of aqueous solutions of sodium alginate in various concentrations to create medicinal hydrogel compositions.

Materials and methods: sodium alginate (Shandong Topsea Seaweed Industrial Co., Ltd., China) was used as a gelling agent. The physicochemical properties of the samples were studied according to generally accepted methods of the State Pharmacopoeia of Ukraine. Rheological studies of experimental samples were carried out using a rotational viscometer «Rheolab QC», by Anton Paar (Austria) with coaxial cylinders C-CC27/SS at a temperature $20-25^{\circ} \mathrm{C}$.

Results and discussion: the rheological profiles of sodium alginate gels have a non-Newtonian type of flow, which makes it possible to characterize them as systems with plastic-viscous properties. Gels with a sodium alginate concentration of $1.5-2 \%$ have the best indicators of yield stress, hysteresis loop area, mechanical stability and dynamic flow coefficients. This indicates a low degree of destruction of the structural grid in the process of mechanical action and the presence of thixotropic bonds.

Conclusions: sodium alginate gel base at $0.5 \%$ and $1 \%$ concentration is less stable under stress compared to sodium alginate bases at $1.5 \%$ and $2 \%$ concentration. The gel base of sodium alginate at a concentration of $2 \%$ has the optimal mechanical stability value.

Keywords: sodium alginate, rheology, structural viscosity, shear stress, mechanical stability.
\end{abstract}

DOI: $10.21303 / 2504-5679.2021 .001732$

\section{Introduction}

In an integrated approach to the problem of local treatment of wounds and burns, one of the main places is given to treatment using semisolid pharmaceutical forms (gels, creams). The course of the wound process is a complex set of protective reactions of the body, the development of which occurs as a response to tissue damage. The body's defense reactions are manifested in the form of destructive and regenerative processes in the wound area and general reactive changes of the body. There are many features, pathways for the course of the wound process and wound healing. However, therapy is determined by the phase and depth of the lesion. It is also necessary to take into account the peculiarities of the course of a particular wound process. This should be taken into account when developing a technology for obtaining wound dressings (applications) used in various fields of medicine.

Recently, much attention has been attracted by materials based on biopolymers, which differ from synthetic polymers in the absence of toxicity and biocompatibility with living systems [1-3]. Among biopolymers, polysaccharides are most widely used, and among them, the most widespread is alginate, which is an anionic polyelectrolyte. Alginates, linear anionic polysaccharides of marine brown algae, are widely used as gelling agents in the food industry, pharmacy, cosmetics, and biotechnology. They form gels in aqueous solutions with the addition of sodium salts or oppositely charged polymers $[4,5]$.

The aim of the work was a comparative study of the main rheological parameters of sodium alginate as a gel-former in various concentrations. The study aimed to determine the dynamic viscosity of alginate gels at various concentrations in solutions. 


\section{Materials and methods}

The objects of the study were sodium alginate made in China and hydrogels prepared on its basis.

The polymer composition based on sodium alginate was prepared according to the method, described in the State Pharmacopoeia of Ukraine [6-8]. The polymer composition consists of sodium alginate (powder) and distilled water. Sodium alginate solutions were prepared in concentrations of $0.5 \%, 1 \%, 1.5 \%$ and $2.0 \%$. A weighed portion of the dry substance was dissolved in distilled water ( $\mathrm{pH}$ 5.6-5.8). To prepare these solutions, a weighed portion of dry polymer (sodium alginate) in an amount of $0.13 \mathrm{~g}, 0.25 \mathrm{~g}, 0.375 \mathrm{~g}, 0.5 \mathrm{~g}$, respectively was taken. Weighed the sample on an Electonic Balance model 6002 analytical balance with an accuracy of $0.01 \mathrm{~g}$ and placed in a volume of distilled water required to obtain a solution of the desired concentration. Then it was stirred with a low-speed stirrer for 10 minutes and kept for 24 hours [9-12].

Rheological studies of experimental samples were performed using a «Rheolab QC» rotary viscometer by Anton Paar (Austria) with coaxial cylinders C-CC27/SS at room temperature $20-25^{\circ} \mathrm{C}$.

A weighed portion of the gel of about $17.0( \pm 0.5) \mathrm{g}$ was placed in a container of the outer stationary cylinder, the required temperature was set, and thermostated the sample for $20 \mathrm{~min}$. Using the software, the experimental conditions were set up: (shear rate gradient (from 0.1 to $350 \mathrm{~s}^{-1}$ ), the number of points on the curve of the sample flow (35), and the duration of measurement at each point (1 sec).

The device allows measuring the tangential shear stress $(\tau)$ in the range of $0.5-3.0 \cdot 10^{4} \mathrm{~Pa}$, the shear rate gradient $\left(\mathrm{Dr} \cdot \mathrm{s}^{-1}\right)$ from 0.1 to $4000 \mathrm{~s}^{-1}$, viscosity $(\eta)$ - from 1 up to $10^{6} \mathrm{~Pa} \cdot \mathrm{s}$ [13-15].

The coefficient of dynamic flow was determined at shear rates of 4.25 and $10.3 \mathrm{~s}^{-1}$, which corresponds to the speed of the palm movement when distributing a semisolid dosage form on the skin surface and the viscosity of the system was determined at shear rates of 25.6 and $148.0 \mathrm{~s}^{-1}$ that modulates the speed of technological processing in the process of its manufacture. Based on these results, the values of the efficiency of the dynamical flow of the system were calculated according to the formulas:

$$
\begin{aligned}
& K_{d 1}=\frac{\eta_{4.25}-\eta_{10.3}}{\eta_{4.25}} \cdot 100 \%, \\
& K_{d 2}=\frac{\eta_{25.6}-\eta_{148}}{\eta_{25.6}} \cdot 100 \%,
\end{aligned}
$$

where $K_{d 1}, K_{d 2}$ - efficiency of dynamical flow; $\eta$ - the effective viscosity at certain shear rates.

For a more complete study of gel samples, the indicators of their mechanical stability $(M S)$ were calculated. It is known that the optimal value of MS is 1.

The value of $M S$ was defined as the ratio of the value of the shear yield stress before destruction $\left(\tau_{1}\right)$ to the value of the shear yield stress after the destruction $\left(\tau_{2}\right)$ by the formula:

$$
M S=\frac{\tau_{1}}{\tau_{2}} .
$$

The study of the viscosity properties makes it possible to assess the thixotropy of systems, to consider the nature of hysteresis loops in the case of their manifestation in systems under deformation loads, and, in general, to analyze the features of the viscous flow of the polymer compositions under study [16-18].

\section{Results}

An integral part of semisolids development is a comprehensive rheological study of model bases, which allows concluding about the effect of excipients on the structural and mechanical parameters of a formulation, on which biopharmaceutical and consumer properties depend, as well as to rationally select the technological parameters of the manufacturing process [19-21].

In the course of the experiment, the structural and mechanical characteristics of sodium alginate gels prepared in various concentrations and their effect on the rheological and organoleptic properties of gels were investigated. 
The studied gels were prepared according to the technology described above. As a result, four samples of the studied gels with a concentration of $0.5 \%, 1 \%, 1.5 \%$, and $2 \%$ were obtained, and the organoleptic evaluation of the samples has been carried out. The organoleptic evaluation of the consistency indicators of the obtained gels is presented in Table $\mathbf{1 .}$

Table 1

Organoleptic evaluation of the alginate gels consistency

\begin{tabular}{cl}
$\begin{array}{c}\text { Concentration of sodium } \\
\text { alginate in solution, } \%\end{array}$ & \multicolumn{1}{c}{ Organoleptic evaluation } \\
\hline $0.5 \%$ & $\begin{array}{l}\text { Colorless, translucent gel, uniform consistency, liquid, very slightly gelatinous, with a specific smell } \\
\text { of sodium alginate, } \mathrm{pH} \text { values }-6.0-6.8\end{array}$ \\
\hline $1 \%$ & $\begin{array}{l}\text { Colorless, translucent gel, uniform consistency, low viscosity, well flowing, slightly gelatinous, with } \\
\text { a specific odor of sodium alginate, } \mathrm{pH} \text { values }-6.0-6.8\end{array}$ \\
& $\begin{array}{l}\text { Colorless, translucent gel, uniform consistency, viscous, well gelatinous, with a specific odor of so- } \\
\text { dium alginate, } \mathrm{pH} \text { values }-6.0-6.8\end{array}$ \\
& $\begin{array}{l}\text { Colorless, translucent gel, uniform consistency, viscous, well gelatinous, with a specific odor of so- } \\
\text { dium alginate, } \mathrm{pH} \text { values }-6.0-6.8\end{array}$
\end{tabular}

As can be seen from Table 1, the viscosity of the samples with sodium alginate increased from $1.5 \%$, so a further increase in the concentration of the gelling agent was impractical.

It has been found that the intensity of the structuring process of alginate gels is in direct proportion to the concentration of the polysaccharide in the solution and is described by a linear equation with a high coefficient of approximation.

The nature of intermolecular interactions in the «sodium alginate-water» system is associated with the course of various processes accompanying dissolution [22-24]. In solution, sodium alginate can exist in the form of aggregates of different sizes with varying degrees of polymerization and water permeability. These factors have a significant impact on the configuration and strength of the formed spatial network of the polysaccharide in water, and, consequently, on the viscosity of the resulting colloidal system.

The next stage of the study was to investigate the effect of sodium alginate concentration on the rheological properties of gels.

The structure strength of the experimental samples was studied by the method of continuous, ever-increasing destruction of the structure, as a function of the shear stress. The determination was carried out by increasing the number of spindle revolutions, reaching a constant shear stress at the maximum number of revolutions, and then decreasing the spindle speed $[25,26]$.

According to the research data, rheograms, reflecting the dependence of the tangential shear stress on the rate of deformation were constructed, which were used to judge the type of flow of the system and the presence of thixotropic properties. When passing from low loads to high and from high to low loads, «ascending» and «descending» flow curves (hysteresis loops) of the samples under study were obtained.

Analyzing the data in Fig. 1, all the samples under study have a non-Newtonian type of flow, which makes it possible to characterize them as systems with plastic-viscous properties. The flow of the samples does not start immediately, but after the application of the deformation stress, the value of which is shown in Fig. 1 and Table 2.

It is known that the presence of hysteresis loops shows that the investigated disperse systems possess thixotropic properties. The significant surface area of the hysteresis loop indicates a sufficient degree of thixotropy of the gel bases. As shown in Fig. 1-3 gel bases with sodium alginate are characterized by a pseudoplastic flow type. The largest area of the hysteresis loop was in the sample of $2 \%$ sodium alginate gel, which indicates the strength of the gel structure. 
We have calculated the coefficient of dynamic flow, which was determined at shear rates of 4.25 and $10.3 \mathrm{~s}^{-1}$. These rate data correspond to the speed of palm movement when a semisolid dosage form is distributed over the skin surface and the viscosities of the system at shear rates of 26.5 and $148.0 \mathrm{~s}^{-1}$, which modulate the processing speed during its manufacturing.

TAU

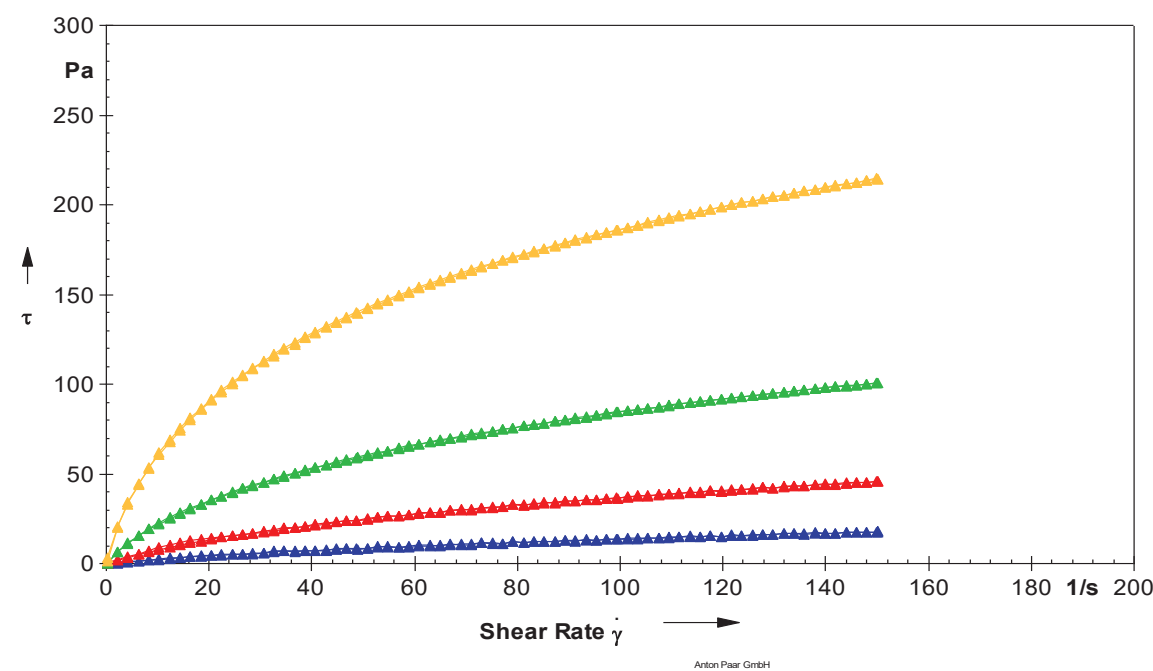

$0.5 \% 1$

CC27/S-SN29766; $\mathrm{d}=0 \mathrm{~mm}$

- $\tau$ Shear Stress

$1.0 \% 1$

CC27/S-SN29766; d=0 mm

- $\tau$ Shear Stress

$1.5 \% 1$

CC27/S-SN29766; d=0 mm

\ $\tau$ Shear Stress

CC27/S-SN29766; d=0 mm

\ $\tau$ Shear Stres

Fig. 1. Curves of the shear stress dependence on the shear rate gradient

Table 2

The values of the structural viscosity and shear stress of gel samples as a function of the shear rate gradient

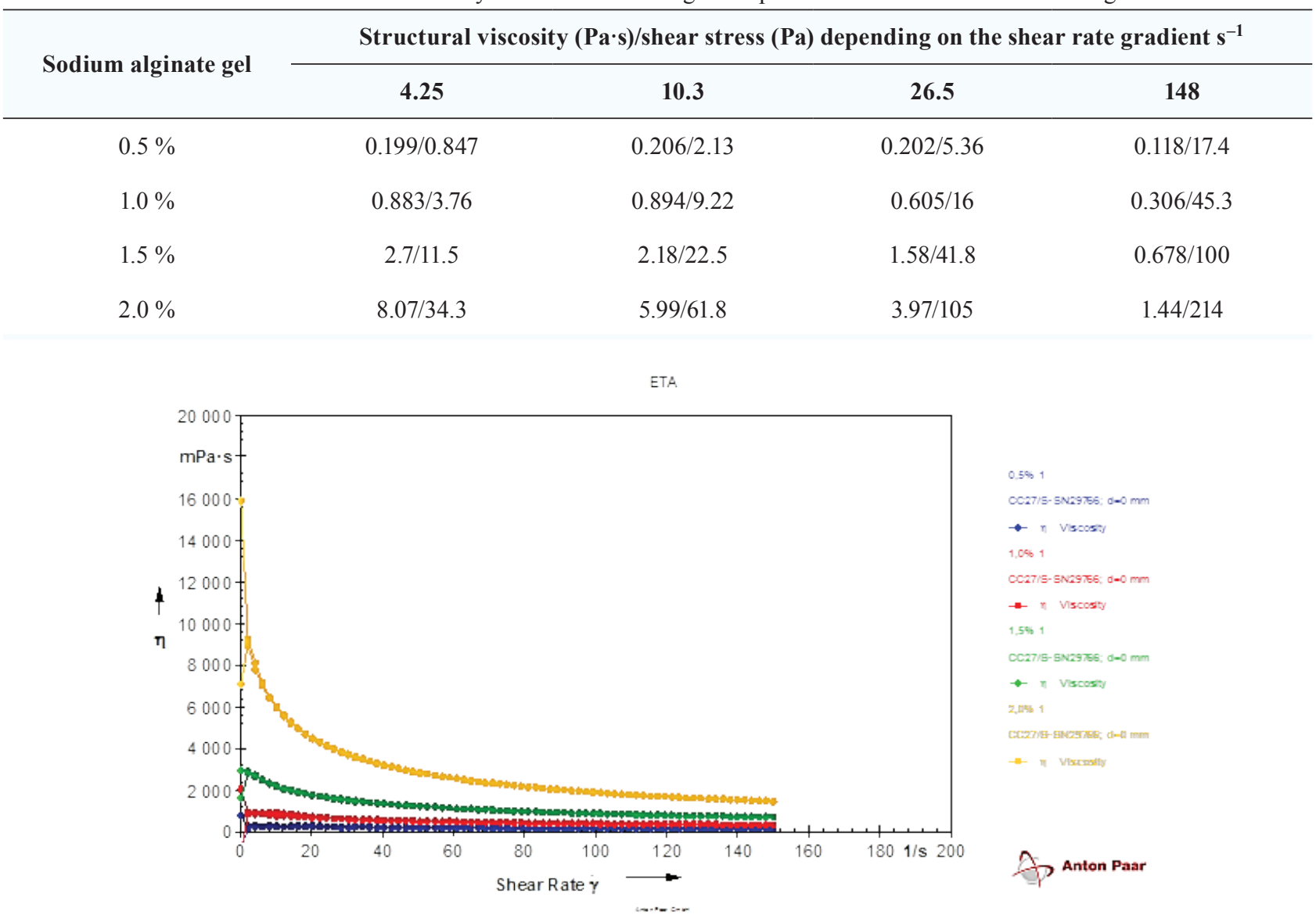

Fig. 2. Curves of the dependence of the structural viscosity of gels on the shear rate gradient 


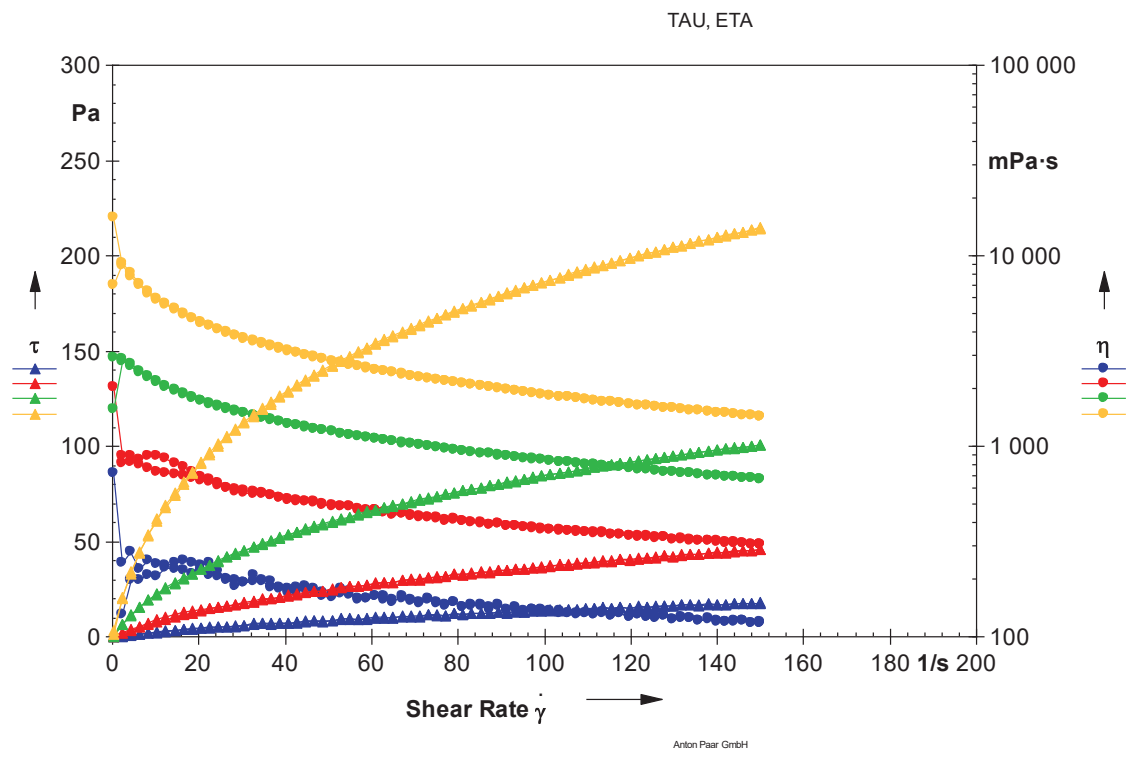

$0.5 \% 1$

CC27/S-SN29766; d=0 mm

- $\tau$ Shear Stress

- $\eta$ Viscosity

$1.0 \% 1$

CC27/S-SN29766; d=0 mm

— $\tau$ Shear Stress

$\rightarrow \eta$ Viscosity

$1.5 \% 1$

CC27/S-SN29766; d=0 mm

- $\tau$ Shear Stress

- $\eta$ Viscosity

$20 \%$

C27/S-SN29766; d=0 mm

$\tau$ Shear Stress

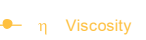

Anton Paar

Fig. 3. Complete rheograms of sodium alginate gels flow

Also, one of the characteristics of the strength of the structure is mechanical stability $(M S)$. $M S$ is an indicator of the degree of destruction of the gel structure in the process of irreversible deformation. For a more complete study of gel samples, the indicators of its mechanical stability were also calculated.

The obtained data were processed and entered in Table 3.

Table 3

The values of the structural parameters of gels at a temperature of $20^{\circ} \mathrm{C}$

\begin{tabular}{ccccccc}
\hline \multirow{2}{*}{ Sodium alginate gel } & \multicolumn{5}{c}{ Indicators } \\
\cline { 2 - 7 } & Yield stress, Pa & Hysteresis loop area, Pa/s & $\boldsymbol{K}_{\boldsymbol{d} \mathbf{1}}$ & $\boldsymbol{K}_{\boldsymbol{d} \mathbf{2}}$ & $\boldsymbol{M S}$ at $\boldsymbol{K}_{\boldsymbol{d} \mathbf{1}}$ & $\boldsymbol{M} \boldsymbol{S}$ at $\boldsymbol{K}_{\boldsymbol{d} \mathbf{2}}$ \\
\hline $0.5 \%$ & 0.070058 & 0.1245 & 3.52 & 41.58 & $0.71-1.04$ & $0.71-1.04$ \\
$1.0 \%$ & 0.49304 & 0.30863 & 1.25 & 49.42 & $1.06-1.22$ & $1.06-1.22$ \\
$1.5 \%$ & 0.23395 & 0.85026 & 19.26 & 57.09 & $1.00-1.03$ & $1.00-1.03$ \\
$2.0 \%$ & 2.4578 & 1.6389 & 25.77 & 63.73 & $1.02-1.04$ & $1.00-1.04$
\end{tabular}

It is known that the optimal $M S$ value is 1 .

As can be seen from the data in Table 3, samples 3 and 4 had rather small values of this indicator, which indicates an insignificant degree of destruction of the structural frame of the studied gels and allowed to suggest the presence of coagulation bonds in the bases, which can be restored after the destruction of the system. This restructuring ability is essential in the production of semisolids. The gel base of sodium alginate at a concentration of $1.5-2.0 \%$ possessed the closest to the optimal $M S$ value, which indicates a low degree of destruction of the structural frame during mechanical action and the presence of thixotropic bonds.

\section{Discussion}

Rheological studies in the development of new drugs make it possible to conclude on the effect of excipients on the physicochemical, biopharmaceutical, and consumer properties, as well as to rationally select the technological parameters of production.

Variations and the effect of alginate concentrations on rheological parameters were illustrated in Table 3 and it has been found that the index of mechanical strength $(M S)$ decreases with 
a decrease in the concentration of sodium alginate. At a constant increase in the shear stress, alginate solutions with different concentrations demonstrated typical behaviour of the non-Newtonian type of flow (Fig. 3), especially in the range of higher shear rates (1-200 s $\mathrm{s}^{-1}$ ). This behaviour occurs due to the alignment of the molecules in the colloidal solution. These results are fully comparable with the results of studies by other scientists $[27,28]$.

To study the phenomenon of hysteresis, which is used to determine the behaviour of polymer macromolecules during the transition from the rheological non-stationary phase to the rheological stationary phase and vice versa, aqueous alginate solutions were tested in both geometries from low to high shear rates, immediately after which a test from high to low was performed. Fig. 1-3 show the hysteresis for the geometry of the «Rheolab QC» rheometer. It has been found that the steepness of the descending curve is higher than the steepness of the ascending one. Based on these results, sodium alginate gels exhibit typical anti-thixotropic behaviour.

Study limitations. Include the impossibility of simultaneous determination of both the yield point (in rotation) and the flow point (in oscillation). These studies allow characterizing the threshold shear stress, above which solid at rest material will begin to flow. This is especially important for the pharmaceutical development of semisolid dosage forms.

Prospects for further research. Oscillation studies with sodium alginate solution, which, in comparison with rotational tests, allow the measurement of viscoelastic solids, and the elastic and viscous parts of the samples can be separated instead of measuring the overall viscosity function. Also, information about how the sample behaves at rest can be obtained, which allows a more accurate characterization of the sample without additional effort.

\section{Conclusions}

Modern, objective methods for studying the properties of polymers have been used: viscometry, standard, and specially developed test methods. The results obtained have shown that, in terms of its structural and mechanical parameters, the gel base of sodium alginate is acceptable for the creation of semisolid dosage forms for topical use.

Sodium alginate gel base at $0.5 \%$ and $1 \%$ concentration is less stable under stress compared to sodium alginate base at $1.5 \%$ and $2 \%$ concentration.

The gel base of sodium alginate in a concentration of $2 \%$ possesses optimal indicators of mechanical stability, which determines in the future good spreadability of the finished semisolid dosage form, as well as its ability to extrusion.

It can be assumed that sodium alginate, together with other biopolymers (as a matrix «depot» for a medicinal product) will achieve a synergistic effect and additional therapeutic action by increasing the biological activity.

From a technological point of view, sodium alginate is an excellent basis for creating multi-component hydrogel compositions.

\section{Conflict of interests}

The authors declare that they have no conflicts of interest.

\section{References}

[1] Kloareg, B., Quatrano, R. S. (1988). Structure of the cell walls of marine algae and ecophysiological functions of the matrix polysaccharides. Oceanography and Marine Biology: An Annual Review, 26, 259-315.

[2] Viswanathan, S., Nallamuthu, T. (2007). Extraction of sodium alginate from selected seaweeds and their physiochemical and biochemical properties. nternational Journal of Innovative Research in Science, Engineering and Technology, 3 (4), 10998-11003.

[3] Sellimi, S., Younes, I., Ayed, H. B., Maalej, H., Montero, V., Rinaudo, M. et. al. (2015). Structural, physicochemical and antioxidant properties of sodium alginate isolated from a Tunisian brown seaweed. International Journal of Biological Macromolecules, 72, 1358-1367. doi: http://doi.org/10.1016/j.ijbiomac.2014.10.016

[4] Campos-Vallette, M. M., Chandía, N. P., Clavijo, E., Leal, D., Matsuhiro, B., Osorio-Román, I. O., Torres, S. (2010). Characterization of sodium alginate and its block fractions by surface-enhanced Raman spectroscopy. Journal of Raman Spectroscopy, 41, 758-763. doi: http://doi.org/10.1002/jrs.2517 
[5] Masuelli, M. A., Illanes, C. O. (2014). Review of the characterization of sodium alginate by intrinsic viscosity measurements. Comparative analysis between conventional and single point methods. International Journal of Biomaterials, $1(1), 1-11$.

[6] Derzhavna Farmakopeia Ukrainy. Vol. 1 (2015). Kharkiv: DP «Ukrainskyi naukovyi farmakopeinyi tsentr yakosti likarskykh zasobiv», 1128.

[7] Council of Europe. European Pharmacopoeia (2016). Strasbourg: Council of Europe. Available at: https://www.coe.int/ en/web/portal/-/the-9th-edition-european-pharmacopoeia-maintaining-high-quality-standards-in-a-dynamic-globalenvironment

[8] The United States Pharmacopeia 39 - NationalFormulary 34 (2016). Washington: The United States Pharmacopeial Convention. Available at: https:/www.worldcat.org/title/united-states-pharmacopeia-the-national-formulary/oclc/933365422

[9] Thakur, V. K., Thakur, M. K. (2015). Handbook of polymers for pharmaceutical technologies. Vol 1. New York. John Wiley \& Sons. doi: http://doi.org/10.1002/9781119041450

[10] Arvidson, S. A., Rinehart, B. T., Gadala-Maria, F. (2006). Concentration regimes of solutions of levan polysaccharide from Bacillus sp. Carbohydrate Polymers, 65 (2), 144-149. doi: http://doi.org/10.1016/j.carbpol.2005.12.039

[11] Graessley, W. (1980). Polymer chain dimensions and the dependence of viscoelastic properties on concentration, molecular weight and solvent power. Polymer, 21 (3), 258-262. doi: http://doi.org/10.1016/0032-3861(80)90266-9

[12] Cheng, R. (1997). On the concentration regimes of a flexible-chain polymer solution. Macromolecular Symposia, 124 (1), 27-34. doi: http://doi.org/10.1002/masy.19971240106

[13] Fu, S., Thacker, A., Sperger, D. M., Boni, R. L., Buckner, I. S., Velankar, S. et. al. (2011). Relevance of Rheological Properties of Sodium Alginate in Solution to Calcium Alginate Gel Properties. AAPS PharmSciTech, 12 (2), 453-460. doi: http://doi.org/ 10.1208/s12249-011-9587-0

[14] Dodero, A., Williams, R., Gagliardi, S., Vicini, S., Alloisio, M., Castellano, M. (2018) Characterization of hyaluronic acid by dynamic light scattering and rheological techniques. AIP conf proc, 1981, 020184. doi: http://doi.org/ $10.1063 / 1.5046046$

[15] Brummer, R., Griebenow, M., Hetzel, F., Uhlmann, R. (2000). Rheological swing test to predict the temperature stability of cosmetic emulsions. Conf proc XXIst IFSCC international congress. Berlin, 476-484.

[16] Sachan, N. K., Pushkar, S., Jha, A., Bhattcharya, A. (2009). Sodium alginate: the wonder polymer for controlled drug delivery. Journal of Pharmacy Research, 2 (8), 1191-1199.

[17] Higgins, J. S. (1979). Polymer conformation and dynamics. Treatise on Materials Science \& Technology, 15, $381-422$. doi: http://doi.org/10.1016/b978-0-12-341815-9.50016-1

[18] Xue, J., Xie, J., Liu, W., Xia, Y. (2017). Electrospun Nanofibers: New Concepts, Materials, and Applications. Accounts of Chemical Research, 50 (8), 1976-1987. doi: http://doi.org/10.1021/acs.accounts.7b00218

[19] Vicini, S., Mauri, M., Vita, S., Castellano, M. (2018). Alginate and alginate/hyaluronic acid membranes generated by electrospinning in wet conditions: Relationship between solution viscosity and spinnability. Journal of Applied Polymer Science, 135 (25), 46390. doi: http://doi.org/10.1002/app.46390

[20] Chandia, N. P., Matsuhiro, B., Vasquez, A. E. (2001). Alginic acids in Lessonia trabeculata: characterization by formic acid hydrolysis and FT-IR spectroscopy. Carbohydrate Polymers, 46 (1), 81-87. doi: http://doi.org/10.1016/s0144$8617(00) 00286-1$

[21] Chandía, N. P., Matsuhiro, B., Mejías, E., Moenne, A. (2004). Alginic acids in Lessonia vadosa: Partial hydrolysis and elicitor properties of the polymannuronic acid fraction. Journal of Applied Phycology, 16 (2), 127-133. doi: http://doi.org/10.1023/ b:japh.0000044778.44193.a8

[22] Lima, A. M. F., Soldi, V., Borsali, R. (2009). Dynamic light scattering and viscosimetry of aqueous solutions of pectin, sodium alginate and their mixtures: effects of added salt, concentration, counterions, temperature and chelating agent. Journal of the Brazilian Chemical Society, 20 (9), 1705-1714. doi: http://doi.org/10.1590/s0103-50532009000900020

[23] Masuelli, M. A. (2014). Mark-Houwink parameters for aqueous-soluble polymers and biopolymers at various temperatures. Journal of Polymer and Biopolymer Physics Chemistry, 2 (2), 37-43.

[24] Pamies, R., Schmidt, R. R., Martínez, M. del C. L., Torre, J. G. de la. (2010). The influence of mono and divalent cations on dilute and non-dilute aqueous solutions of sodium alginates. Carbohydrate Polymers, 80 (1), 248-253. doi: http://doi.org/ 10.1016/j.carbpol.2009.11.020

[25] Gladukh, Ie., Grubnik, I., Kukhtenko, H. (2017). Structural-Mechanical Studies of Phytogel «Zhivitan». Journal of Pharmaceutical Sciences and Research, 9 (10), 1672-1676.

[26] Kukhtenko, H., Gladukh, Ie., Kukhtenko, O., Soldatov, D. (2017). Influence of Excipients on the Structural and Mechanical Properties of Semisolid Dosage Forms. Asian Journal of Pharmaceutics, 11 (3), 575-578. 
[27] Wunderlich, T., Stelter, M., Tripathy, T., Nayak, B. R., Brenn, G., Yarin, A. L. et. al. (2000). Shear and extensional rheological investigations in solutions of grafted and ungrafted polysaccharides. Journal of Applied Polymer Science, 77 (14), $3200-3209$. doi: http://doi.org/10.1002/1097-4628(20000929)77:14<3200::aid-app220>3.0.co;2-9

[28] Sanchez, E. M. (2005). Impact of Particle Morphology on the Rheology of PCC-Based Coatings, 117.

Received date 10.02.2021

(C) The Author(s) 2021

Accepted date 21.03.2021

This is an open access article under the CC BY license

Published date 29.03.2021

(http://creativecommons.org/licenses/by/4.0).

How to cite: Gladukh, I., Podorozhna, M. (2021). Study of structural and mechanical properties of sodium alginate gels. EUREKA: Health Sciences, 2, 82-89. doi: http://doi.org/10.21303/2504-5679.2021.001732 